\title{
Schöne Aussichten
}

Wir Zahnärzte haben uns daran gewöhnt, in regelmäßigen Abständen mit Gesetzen konfrontiert zu werden, die unsere freie Berufsausübung immer weiter einschränken. Die Blaupause den Masterplan - hat bereits Ende der 70er Jahre der Ortskrankenkassentag der Allgemeinen Ortkrankenkassen (AOK) in einem Weißbuch geliefert, in dem das Ziel der Brechung des Kartells der Leistungsanbieter ausgegeben wurde. Hat man sich zunächst in den 70er und 80er Jahren auf reine Kostendämpfungsgesetze beschränkt, so wurde in den 90ern der Weg der strukturellen Änderung hin zum staatlich organisierten und verwalteten Gesundheitssystem mit der Einführung von strikter Budgetierung eingeschlagen. Seitdem hat eine Kettengesetzgebung wechselnder Regierungen dafür gesorgt, dass unser Gesundheitssystem nicht zur Ruhe kommt. Die Vorstellung, Gesundheit zentralistisch - und damit bürgerfern - zu organisieren, ist beherrschende Ideologie der führenden Gesundheitspolitiker. Subsidiarität und das gewachsene Arzt-Patienten-Vertrauensverhältnis bleiben dabei auf der Strecke.

\section{Was wird 2017 bringen?}

Was ist auf diesem für die freie Berufsausübung verhängnisvollen Weg für das nächste Jahr zu erwarten? Das SVSG, liebevoll von seinen Verfassern Selbstverwaltungsstärkungsgesetz genannt, ist in seinen abstrusesten Werkzeugen entschärft worden. Es bleibt trotzdem genug zusätzliche Aufsicht, um die Spielräume der Selbstverwaltung weiter nachhaltig einzuengen. Speziell die vorgesehene obligatorische namentliche Zwangsabstimmung, um aus der Gruppen- eine Einzelhaftung zu machen sowie die De-facto-Aufhebung des Haushaltsrechts der Vertreterversammlung führen von der Rechts- zur Fachaufsicht. Hier muss mit allen Kräften versucht werden, den Selbstverwaltungsspielraum zu erhalten.

Die Umsetzung des Antikorruptionsgesetzes liegt nun in den Händen der Staatsanwaltschaften. Die Kriminalisierung einer Berufsgruppe war von der Politik gewollt, und im gesamtgesellschaftlichen Diskurs war niemand bereit, sich auf unsere Seite zu stellen. Die Körperschaften können nur noch die neue Rechtslage erklären und vor möglichen Fallgruben warnen. Das letzte Wort werden die Gerichte haben. Der Freie Verband steht mit seiner Rechtsberatung für alle Mitglieder zur Verfügung.

Die Novelle der GOÄ hängt auch weiterhin wie ein Damoklesschwert über der Zahnärzteschaft. Wenn die Ärzte bereit sind, Rechte gegen Geld einzutauschen, wird das nicht ohne schwerwiegende Folgen für die Zahnärzteschaft bleiben. Es gilt, das Ziel fest im Blick zu behalten: die Möglichkeit der freien Vertragsgestaltung zwischen Arzt und Patient herzustellen.

Die wettbewerbsverzerrende Einführung zahnarztgleicher MVZs wird den Markt im ambulanten zahnmedizinischen Bereich auf Dauer hin zu Großstrukturen mit rein ökonomischem Hintergrund verändern. Nur dort, wo es sich für Kapitalgeber nicht lohnt, darf die Kleinpraxis die Versorgung in der Fläche sicherstellen. Hier gilt es, den fairen Wettbewerb wiederherzustellen und gleichzeitig Modelle zu entwickeln, die flexible Niederlassungsmöglichkeiten, ökonomische Risikobegrenzung und sichere Rahmenbedingungen ermöglichen, um auch die kommende Zahnarztgeneration für die freie Berufsausübung zu begeistern.

Die Bundestagswahl im nächsten Jahr wirft ihre Schatten voraus. Jüngst hat Bundeskanzlerin Angela Merkel ihre Bereitschaft für eine erneute Amtszeit erklärt - nicht völlig überraschend. Bei der Bundesversammlung der Bundeszahnärztekammer hat Minister Gröhe sein Bekenntnis zum dualen Versicherungssystem abgegeben. Man wird sehen, was diese Bekenntnis nach der Wahl in welcher Regierungskonstellation wert sein wird. Alle sonstigen momentan im Bundestag vertretenen Parteien haben sich klar zur Bürgerversicherung mit Einheitsversorgung bekannt. Das Motto der Vereinheitlicher lautet: Wenn es schon nicht allen gleich gut gehen kann, dann soll es wenigstens allen gleich schlecht gehen. Hier gilt es, unsere Vorstellungen von Subsidiarität und Selbstbestimmung in die Debatte einzubringen. Die Zahnärzteschaft ist gut beraten, auf alle Möglichkeiten vorbereitet zu sein. Insbesondere diejenigen zur unabhängigen, staatsfernen Selbstorganisation müssen geprüft werden.

\section{Mit einer Stimme sprechen}

Liebe Kolleginnen und Kollegen, die gesundheitspolitische Lage für die Zahnärzteschaft wird auch 2017 und darüber hinaus nicht leichter werden. Aber im Gegensatz zu den Ärzten gibt es bei uns einen Hoffnungsschimmer: Der Berufsstand hat begonnen, nach außen wieder mit einer Stimme zu sprechen. Alle maßgeblichen Organisationen haben sich auf die Grundzüge einer modernen PAR-Therapie geeinigt, die es nun umzusetzen gilt. Dass unterschiedliche Vorstellungen in diesem Prozess zusammengeführt werden konnten, macht Mut für die Zukunft. Bei allen dunklen Wolken ist das ein Hoffnungsstreif mit schöner Aussicht. Wir haben schwierige Verhandlungen zur Umsetzung einer im Gemeinsamen Bundesausschuss (G-BA) bereits beschlossenen Richtlinie zur Qualitätssicherung vor uns. Nur ein geschlossener Berufsstand hat überhaupt eine Chance, seine Vorstellungen von Freiberuflichkeit umsetzen zu können. Daran lassen Sie uns alle weiter gemeinsam arbeiten.

Ich wünsche allen ein gesundes und erfolgreiches Jahr 2017.

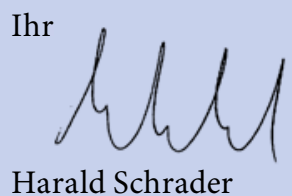

Harald Schrader

FVDZ-Bundesvorsitzender 